\title{
The Effect of Contralateral Signal on Distortion Product Otoacoustic Emissions and Psychophysical Tuning Curves at 1 and $2 \mathrm{kHz}$
}

\author{
A. WICHER* \\ Institute of Acoustics, Faculty of Physics, Adam Mickiewicz University, Umultowska 85, 61-614 Poznań, Poland
}

\begin{abstract}
The main aim of this work was to determine the influence of contralateral stimulation (CS) on the psychophysical tuning curves (PTCs) and distortion product otoacoustic emissions (DPOAEs). PTCs and DPOAEs were measured in two modes: in the presence or absence of CS. The contralateral signal was a wideband noise (bandwidth $0.2-10 \mathrm{kHz}$ ) at a level of $50 \mathrm{~dB}$ sound pressure level (SPL). The primary tones $\left(F_{1}\right.$ and $\left.F_{2}\right)$ were presented at levels of $L_{1}=60 \mathrm{~dB}$ SPL, and $L_{2}=50 \mathrm{~dB}$ SPL. The signal frequency used in the measurements of the PTC was $1 \mathrm{kHz}$ or $2 \mathrm{kHz}$. For both PTC signal frequencies the CS significantly reduces the sharpness of the PTCs. The average change in level of DPOAE under the influence of CS throughout the whole range of frequencies takes an effect of suppression. The CS has a significant effect on decreasing the value of the quality factor $\left(Q_{10 \text { Roex }}\right)$ of PTCs $(F(1,9)=19.36, p=0.002)$. The CS caused a decrease in the level of DPOAE in $88 \%$ of cases. The maximum suppression of the DPOAE level occurs for the $F_{2}$ frequency from $1 \mathrm{kHz}$ to $2 \mathrm{kHz}$.
\end{abstract}

DOI: 10.12693/APhysPolA.123.1001

PACS: 43.64.Jb, 43.66.Dc

\section{Introduction}

Contralateral stimulation (CS) of the auditory system is a method of examination which enables analysis of how the efferent part of the auditory pathway functions [1-10]. The outer hair cells (OHCs) are largely responsible for the active processes in the cochlea; and they have an effect on the cochlea's micromechanics [11]. The outer hair cells are connected to the efferent system via the medial olivocochlear (MOC) system. Hence contralateral stimulation by means of the MOC system has an effect on the functioning of the OHCs [4]. Stimulation of the efferent system has an effect on changes in the levels of otoacoustic emission (OAE) $[4,7,9,10,12-15]$; on reductions in the masking effect for tone-burst signal types; and on the intelligibility of speech when presented at a background of noise [16-18]. Kumar and Vanaja [16], and Kim et al. [17] suggest that the functioning of the MOC system supports the process of speech perception against an interfering noise. However, Wagner et al. [18] stated that speech-in-noise intelligibility did not correlate with MOC activity.

The distortion products OAE (DPOAE) are characterized by the appearance of non-linear distortions, and the highest registered level for these distortions occurs for frequency $F_{\mathrm{DP}}=2 F_{1}-F_{2}$, where $F_{1}$ and $F_{2}$ denote the frequencies of primary tones $[9,19-21]$.

The DPOAE is often used in research on CS and in investigations of the MOC system's functioning. Contralateral stimulation causes a reduction in the DPOAE level (from 0.5 to $2 \mathrm{~dB}$ ) $[9,22,23]$. Reduction in the level

*e-mail: awaku@amu.edu.pl of OAE is known as the suppression phenomenon [4, 24]. Contralateral change in the value of DPOAE depends on both the type and level of CS. The most effective suppressor is the wideband noise (WBN) $[14,25]$. In the case of DPOAE fine structure, most suppression is observed in the maximum range of DP-gram, whereas in the minimum range the suppression is lower, or sometimes the enhancement effect of DPOAE signals can be observed [8, 13, 26-28].

On the whole, it can be stated that stimulation of the efferent system inhibits the functioning of the cochlear amplifier [29] which in turn can lead to a change in masking effect in the presence of CS [5, 30,31].

Auditory masking is commonly used to assess the frequency selectivity of the auditory system [32-35]. The masking phenomenom can be determined by measuring the psychophysical tuning curve (PTC) [36-40]. Measurement of PTC is achieved by determining the masker level at the auditory threshold of the tonal signal, at the level of $10 \mathrm{~dB}$ sensation level (SL) for various masker center frequencies. For individuals with normal hearing, the minimum (tip) of the PTC corresponds to the frequency of the tonal signal, and the PTC curve has a steep slope of the low- and high-frequency sides. In cases of damaged $\mathrm{OHCs}$, a reduction of the frequency selectivity is usually observed [41, 42$]$. It reduces the dynamic range of PTC considerably and decreases the PTC slope of the low- and high-frequency sides $[43,44]$. One of the parameters characterizing PTCs is the value of $Q_{10}$, defined as the characteristic frequency (CF) divided by the PTC bandwidth measured at $10 \mathrm{~dB}$ above the PTC tip. Reduction of the frequency selectivity results in (among other things) a lower $Q_{10}$ value.

Until now there has been little research conducted on the effect of CS on PTC. Kawase et al. [30] and Vinay and Moore [5] have shown that using a contralateral sig- 
nal causes changes in the slopes of the low- and highfrequency sides of PTC and the value of $Q_{10}$. Kawase et al. [30] have demonstrated that using CS in the form of WBN leads to a reduction in masker level at the threshold for frequencies below CF. This research was carried out for a test tone frequency of $2 \mathrm{kHz}$. Then Quaranta et al. [31] showed that for the test tone frequency $f=1 \mathrm{kHz}$, the value of $Q_{10}$ increased during contralateral stimulation, whereas for $f=4 \mathrm{kHz} Q_{10}$ it was reduced.

Vinay and Moore [5] demonstrated that for signal frequencies of $2 \mathrm{kHz}$ and $4 \mathrm{kHz}, \mathrm{CS}$ significantly reduced the masker level at the threshold on both the low and high-frequency sides of the PTCs, and the $Q_{10}$ values decreased significantly. For the lower signal frequencies $(0.5 \mathrm{kHz}$ and $1 \mathrm{kHz})$, the masker level required for the threshold on the low-frequency sides of the PTCs increased with CS, and the $Q_{10}$ values increased significantly. This would suggest that in the lower frequency bands the efferent system causes an increase in frequency selectivity, whereas in the higher band $(2-4 \mathrm{kHz})$, frequency selectivity is reduced.

To determine PTCs, the method used involves establishing the level for the band of noise that is required to just mask the test tone, for several masker center frequencies [45]. However, this method is rather timeconsuming and determining one PTC can take around 90 minutes. Sęk et al. [39] and Sęk and Moore [46] worked on a method of PTC measurement which involved continually sweeping band of noise. This method, known as the FAST-PTC method for determining PTC, yields very similar results to the classic method, and yet shortens research time to a few minutes. Furthermore, the authors provided the optimal values of the sweeping band of noise which yield results comparable to the results derived from using the classic method. For subjects with normal hearing, the tip of the PTC is located close to the signal frequency. PTCs can be used for estimation of the auditory filters because the frequency characteristic of the auditory filter can be obtained by inverting the PTC [40]. The squared frequency response of the auditory filters can be approximated by the rounded exponential function (Roex) [40, 47]. This approximation means that the tip of the PTC can be estimated, as can the slopes of the low and high frequency "tail" of the PTC and also the $Q_{10}$ value.

The effect of the CS can be seen most clearly in the reduced level of OAE in the wide frequency range [15], which should be reflected by an increase in the $Q_{10}$ value of PTC as well as in the whole frequency range. However, this has not been confirmed by research results $[5,31]$.

Bearing in mind the divergent values of $Q_{10}$ for various frequencies of the test tone given in the literature [5], research was carried out to determine the value of $Q_{10}$ for PTC, both in the presence and absence of con- tralateral wideband noise, for two test tone frequencies, $1 \mathrm{kHz}$ and $2 \mathrm{kHz}$.

The aim of this work was to describe the effect of CS on the value of $Q_{10}$ by using the so-called FAST-PTC method of measuring PTC. In order to confirm a suppression effect of the OAE in the wide frequency range, a measurement of DPOAE was also performed, in the presence of CS, to determine changes in the level of DPOAE components at the frequencies $F_{\mathrm{DP}}=2 F_{1}-F_{2}$. An assumption was made: that if in a wide frequency range a suppression effect dominates [8, 15, 28], it should then result in reducing the $Q_{10}$ value in this frequency range, in view of the contralateral stimulation.

\section{Materials and methods}

\subsection{Subjects}

Ten normal-hearing subjects participated in this study. Their ages ranged from 19 to 24 years. The tonal audiometry, determined by means of a clinical audiometer (AC 40 Interacoustics), comprised of measurements of hearing thresholds at standard frequencies from $125 \mathrm{~Hz}$ to $8000 \mathrm{~Hz}$. Subjects had no history of ear diseases and they had hearing thresholds at or better than $15 \mathrm{~dB}$ hearing level (HL) in both ears. The middle ear testing performed by means of a Homoth tympanometer showed normal middle ear function for all subjects.

\subsection{Measurement of PTCs}

PTCs were measured in two modes: in the presence or absence of CS. A narrowband noise masker whose center frequency was swept from below to above the signal frequency was used in the measurements of the PTC. The masker level was changed by the subjects during the investigations. Sęk and Moore [46] developed the Sweeping PTC (SWPTC) software when the fast method for PTC determination was implemented. The option of additional CS was also added to this software. The contralateral signal was a WBN (bandwidth $0.2-10 \mathrm{kHz}$ ) at the level of 50 SPL. SWPTC software was installed on a laptop computer with a good-quality sound card (Sound Blaster X-Fi Xtreme)

A pure-tone signal and a narrowband noise masker were presented to the same ear. The tonal signal was presented at a sensation level (SL) of $10 \mathrm{~dB}$, and was pulsed on and off in a regular manner. Each tone pulse and the gap between successive pulses lasted $200 \mathrm{~ms}$. The signal frequency was $1 \mathrm{kHz}$ or $2 \mathrm{kHz}$. The masker bandwidth was fixed at $20 \%$ of the masker center frequency. The masker level was increased whenever the subject pressed the space bar to indicate that the signal was audible, and was decreased whenever the spacebar was released. The initial level of the masker was $50 \mathrm{~dB}$ SPL. The rate of changes of masker level was $2 \mathrm{~dB} / \mathrm{s}$.

Measurements were repeated three times. In the contralateral mode, the CS was presented continuously during PTC measurement. 


\subsection{Measurement of DPOAEs}

DPOAEs were measured using the Tucker-Davis Technologies (TDT) System 3. As with the PTC measurement, two modes of the stimuli were used in the investigations (with and without the accompaniment of an additional wideband noise to the contralateral ear). The primary tones at frequencies $F_{1}$ and $F_{2},\left(F_{2} / F_{1}=1.22\right)$ were generated using a 24-bit digital real-time signal processor RP2.1 at $44.1 \mathrm{kHz}$ sampling rate. The $F_{2}$ frequency ranged from $0.8 \mathrm{kHz}$ to $6 \mathrm{kHz}$. Signals were sent to the headphone buffer (HB7). Finally, signals from the output of the HB7, via an insert earphone (Etymotic Research, ER-2) were delivered to the ear canal. A low noise microphone (ER 10B + ) inserted into the ear canal in a soft plastic ear tip acquired the sound pressure waveform. The contralateral signal was a wideband noise (bandwidth $0.2-10 \mathrm{kHz}$ ). This CS was generated via an independent channel (RP2.1, HB7 and ER-2) at the level of 50 SPL and delivered to the contralateral ear. The levels of the primary tones and the contralateral signal were calibrated by low-noise probe microphones $($ ER-10B + ) placed in the external ear canals, before basic measurements. Owing to this, it was possible to ensure that an appropriate level for the primary tones and the contralateral signal was maintained each time the stimulus was used. It should be stressed that the processes of all the signal generation and acquisition were fully synchronized by the TDT's triggers system.

Primary tones were presented at the level of $L_{1}=$ $60 \mathrm{~dB}$ SPL, and $L_{2}=50 \mathrm{~dB}$ SPL. The generation and acquisition of the DPOAE signal lasted 6 seconds. It consisted of a 3-second acquisition in the absence of CS and a 3 -second recording in the presence of CS. A coherent averaging technique was used in the experiment. Measurements were made for all $F_{2}$ frequencies without changing the probe position in the ear canal to minimize its possible influence on the DPOAEs. The level of the noise floor was estimated as an average of the amplitude of 20 spectral lines adjacent to the $F_{\mathrm{DP}}$ component. The frequency resolution of the Fast Fourier Transform (FFT) was $0.17 \mathrm{~Hz}$.

A bandpass filtering ( $20 \mathrm{~Hz}$ constant bandwidth, centered at frequency $F_{\mathrm{DP}}$ ) of the recorded waveform was performed and the $\mathrm{F}_{\mathrm{DP}}$ component level changes versus time were determined. The DPOAE levels with and without CS were determined as mean values in time intervals $(1.5 \mathrm{~s})$ which excluded a transient interval and comprised relatively steady level values of the DPOAE temporal record. The $F_{\mathrm{DP}}$ component was accepted as a significant response when the signal-to-noise ratio of the DPOAE exceeded $6 \mathrm{~dB}$. The values of the DPOAE level changes $\left(\Delta L_{\mathrm{DP}}\right)$ were defined as a difference between the mean DPOAE levels with and without contralateral noise.

\section{Results}

\subsection{Analysis of the parameters of the PTCs}

Figure 1 shows an example of the PTC approximation by means of the Roex procedure [46] for situations with-

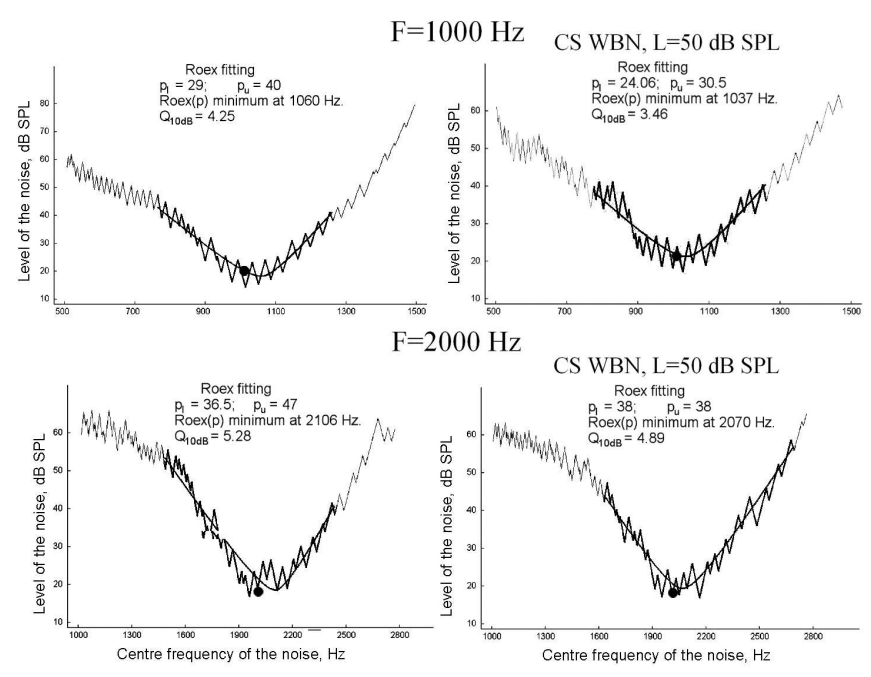

Fig. 1. An example functions of PTC for situations without CS (left column) and with CS (right column), at the signal frequencies of $1 \mathrm{kHz}$ and $2 \mathrm{kHz}$. Black circle indicates the level and the frequency of the signal. The thin, continuous line shows the data from the experiment, whereas the thicker line shows the course of the Roex function using the method of the least-squares error fit. The thick, jagged line shows data used for Roex function fitting.

out CS (left column) and with CS (right), at the signal frequencies of $1 \mathrm{kHz}$ and $2 \mathrm{kHz}$.

The thin, continuous line shows the data from the experiment, whereas the thicker line shows the course of the Roex function using the method of the least-squares error fit [40]. The parameters of every Roex function included filter quality $\left(Q_{10 \text { Roex }}\right)$, the PTC tip value $\left(\operatorname{Min}_{\mathrm{PTC}}\right)$, a slope of the low frequency sides $\left(p_{\text {LRoex }}\right)$, as well as the high-frequency sides $\left(p_{\text {URoex }}\right)$. The results also made it possible to determine the value of the equivalent rectangular bandwidth $\left(E R B_{\text {Roex }}\right)$. In Figure 1 it is clear that the minimum Roex function does not match up with the value of the frequency of the tonal signal and has slightly higher value. This is an effect which is described in the literature [39] and it is caused by the noise-band sweeping (from low to high frequencies).

A two-way within-subjects analysis of variance (ANOVA) was carried out on the $Q_{10 \text { Roex }}$ values, with the factors "frequency" (1000, 2000) and "contralateral noise" (on, off). A statistical analysis was performed using Statistica ver. 9.1 software (StatSoft Inc. 1984-2010). The main effect of contralateral noise was statistically significant $(F(1,9)=19.36, p=0.002)$. The effect of frequency was also significant $(F(1,9)=19.65, p=0.002)$. The interaction of frequency and contralateral noise (Fig. 2) was not statistically significant $(F(1,9)=0.47, p=0.512)$.

For all signal frequencies the CS significantly reduces the sharpness of the tuning measured by the $Q_{10 \text { Roex }}$ parameter. The highest decrease in the $Q_{10 \text { Roex value was }}$ observed for $f=2000 \mathrm{~Hz}$. 


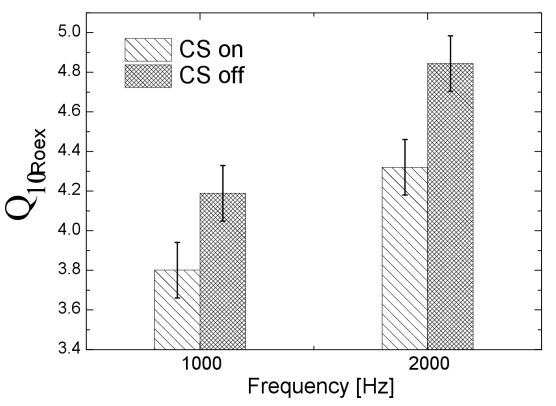

Fig. 2. The average values of $Q_{10 \text { Roex }}$ for signal of frequency $1 \mathrm{kHz}$ and $2 \mathrm{kHz}$, with and without contralateral stimulation. Vertical bars indicate a $95 \%$ confidence interval.

Next, a two-way within-subjects ANOVA on the location of the minimum PTC $\left(\operatorname{Min}_{\mathrm{PTC}}\right)$ showed that the effect of CS was not statistically significant $(F(1,9)=$ $0.011, p=0.919)$. The remaining parameters describing the Roex function are the slopes of the low- and highfrequency sides. The slope of the low-frequency side ( $\left.p_{\text {LRoex }}\right)$ decreased when the CS was present and the difference was statistically significant $(F(1,9)=6.052$, $p=0.015)$. However, the slope of the high-frequency side $\left(p_{\text {URoex }}\right)$ did not change significantly in the presence of CS $(F(1,9)=3.561, p=0.061)$. Therefore, reductions in the value of $Q_{10 \text { Roex }}$ were largely caused by changes in the slope on the low-frequency side of PTCs.

\subsection{DPOAE level changes}

For every subject, measurements of the DPOAE level $\left(L_{\mathrm{DP}}\right)$ were taken, both in the presence and absence of contralateral noise, at the frequencies of $F_{2}$ from $845 \mathrm{~Hz}$ to $6202 \mathrm{~Hz}$.
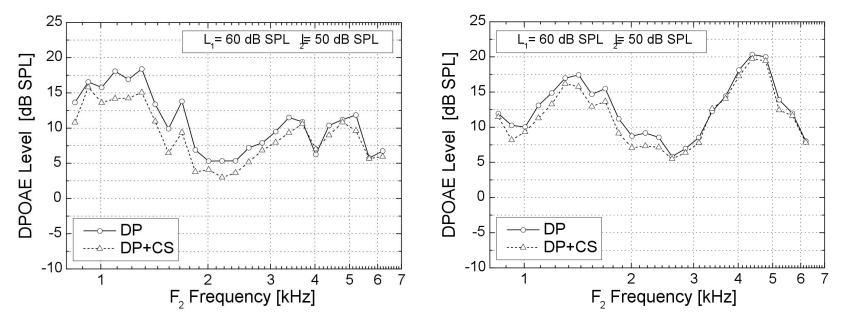

Fig. 3. Examples of DP-grams for 2 participants. The empty circles denote the values of the DPOAE level $\left(L_{\mathrm{DP}}\right)$ derived without $\mathrm{CS}$, whereas the empty triangles show them with CS.

Figure 3 shows example DP-grams for 2 participants. The empty circles denote the values of $L_{\mathrm{DP}}$ derived without CS, whereas the empty triangles show them with CS. It should be emphasized that for the majority of frequencies $F_{2}$, lower values of $L_{\mathrm{DP}}$ were obtained with $\mathrm{CS}$. The DP-gram functions for each participant vary, which has been confirmed in many other studies on DPOAE for people with normal hearing [19]. This is why in further analysis of the results, relative changes in DPOAE $\left(\Delta L_{\mathrm{DP}}\right)$

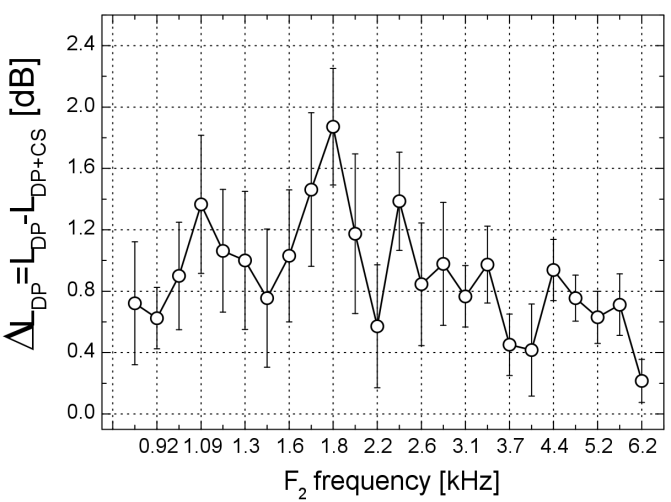

Fig. 4. An average values of relative changes in DPOAE $\left(\Delta L_{\mathrm{DP}}\right)$ as the function of the $F_{2}$ frequency. The vertical bars denote one standard error.

are described as a difference between the value levels without $\mathrm{CS}\left(L_{\mathrm{DP}}\right)$ and value levels with $\mathrm{CS}\left(L_{\mathrm{DP}+\mathrm{CS}}\right)$. Figure 3 demonstrates that $\Delta L_{\mathrm{DP}}$ reached positive values in the range of local maximum DP-gram function and in those ranges where DP-gram is a constant, increasing, or decreasing function of frequency. In turn, with the local minimum function of DP-gram it is possible to observe even insignificant increases in the value level of $L_{\mathrm{DP}+\mathrm{CS}}$. This effect has also been observed by other researchers [8-10, 26, 28, 48, 49].

Figure 4 shows the average values of $\Delta L_{\mathrm{DP}}$ as the function of the $F_{2}$ frequency. The average change in level of DPOAE under the influence of CS throughout the whole range of frequencies takes on a positive value (an effect of suppression) and hovers between around $0.3 \mathrm{~dB}$ for the highest frequencies and about $2 \mathrm{~dB}$ for $F_{2}$ frequencies in the $2 \mathrm{kHz}$ range. The vertical bars denote one standard error. It should be added that examples of increases in the level of DPOAE were also recorded with CS, but they only accounted for $12 \%$ of all the cases. It is therefore possible to state that, for the whole range of the $F_{2}$ frequency, in $88 \%$ of all cases the suppression effect occurs for the acquired components of DP-gram.

\section{Discussion}

Contralateral stimulation utilized in OAE measurements is an objective and non-invasive method which enables the efferent parts of the auditory system to be investigated. In the present study, results of research into DPOAE and PTC in the presence of broadband contralateral signal have been presented. The results of the DPOAE investigation show a reduction in the $2 F_{1}-F_{2}$ component level in the presence of CS, in the whole range of frequencies analyzed (from $845 \mathrm{~Hz}$ to $6202 \mathrm{~Hz}$ ). The average change in the level of DP reached values lower than $2 \mathrm{~dB}$ (in the frequency range of around $2 \mathrm{kHz}$ ). It has to be stressed that this unambiguous effect of DPOAE suppression is observed when CS is a WBN. It is then that the suppression effect is at its greatest. In cases where noise bands or tonal signals are applied, change in the level of otoacoustic emissions is either less or does 
not occur at all $[12,14,25,50]$. Assuming that the average value for the sound pressure level of the DPOAE is no higher than a few $\mathrm{dB}$, the change in the level in the presence of CS comes to several dozen percent of acquired OAE. Above $2 \mathrm{kHz}$ the value of $\triangle D P$ decreases along with the increase in the frequency $F_{2}$ (see Fig. 4), which is in agreement with the results presented by other authors [15].

Measurements of DPOAE did not include frequency ranges below $800 \mathrm{~Hz}$ because of the high level of background noise, even though averaging was applied and the measuring was performed in a soundproof booth. For the investigation of DPOAE and PTC, the frequency range $1-2 \mathrm{kHz}$ was the most significant.

As was mentioned earlier, Vinay and Moore [5] demonstrated that up to the value of $1 \mathrm{kHz}$ of the test tone, an increase in $Q_{10}$ in the presence of CS was observed, whereas from the value of $2 \mathrm{kHz}$ there was a decrease in the value of $Q_{10}$ in the presence of CS. A similar dependence was obtained by Quaranta et al. [31] when comparing the difference in the values of $Q_{20}$ for PTC determined for $1 \mathrm{kHz}$ and $4 \mathrm{kHz}$. However, the results presented by Quaranta et al. [31] have to be treated with some care because a tonal signal was used as a masker. It is well known that using a tone as a masking signal when determining PTC leads to a beating phenomenon which is a factor that can have an effect on the research results $[5,38,51]$. It should also be added that Vinay and Moore [5] and Quaranta et al. [31] applied a 1/3th-octave wide band of noise as a contralateral signal.

Guinan and Gifford [3] used invasive procedures on cats to measure tuning curves (TC) in neurons. They claimed that electrical stimulation of the efferent system causes, in most cases, a reduction in the value of $Q_{20}$ in neurons. However, for characteristic frequencies below $2 \mathrm{kHz}$ the opposite effect was observed in some cases, namely an increase in the value of $Q_{20}$. Consequently, two effects that contradict each other have been observed: the partly confirmed results of research on TC [3] and PTC [5, 30]. Such contradictory results are not observed when measuring changes in the level of OAE in the presence of CS. The results in this study (Fig. 4) as well as results published by other researchers [15] demonstrate the existence of the suppression effect in the whole frequency range $0.8-6 \mathrm{kHz}$. Assuming that a decrease in the level of otoacoustic emission causes a decrease in the effect of nonlinear functioning of the cochlea, one should expect a decrease in the $Q_{10}$ value of PTC for analyzed frequencies, which was confirmed in this paper.

This research, which used broadband contralateral signal, confirmed the hypothesis that there would be a decrease in the value of $Q_{10 \text { Roex }}$ for the frequencies from $1 \mathrm{kHz}$ and $2 \mathrm{kHz}$ (Fig. 2). The larger decrease in $Q_{10 \mathrm{Roex}}$ influenced by $\mathrm{CS}$ was observed for the frequency $2 \mathrm{kHz}$ $(10 \%)$, and this $Q_{10 \text { Roex }}$ reduction is consistent with the highest DPOAE suppression for the $2 \mathrm{kHz}$ frequency region. Decrease in the level of DP in the frequency range $1-2 \mathrm{kHz}$ reached $1.2 \mathrm{~dB}$ on average, whereas for the fre- quency $2 \mathrm{kHz}$ it reached a maximum average value of $1.8 \mathrm{~dB}$.

It should be emphasized that the $Q_{10}$ reduction is closely connected with a statistically significant decrease in the slope on the PTC low-frequency side during CS. Guinan and Gifford [3] showed that electrical stimulation of the MOC system raised the thresholds of fibers for tones at the CF. The contralateral stimulation evoked a decrease in the slope of the low-frequency side of the PTC [52].

Comparing the results of this study with those published by Vinay and Moore [5] the difference in the change of the $Q_{10}$ values during CS should be stressed. In this study the reduction of $Q_{10 \text { Roex }}$ was obtained for both tested frequencies. These results are in line with the data presented by Guinan and Gifford [3] who showed in most cases, a reduction in the quality values of the TC, especially for the medium- and low-spontaneous rates of the auditory-nerve fibers. Physiological findings obtained in animals show that for cats, the MOC system has different patterns of innervation along the length of the cochlea [1,53]. The largest innervation corresponds to the $\mathrm{CF}$ region near $3 \mathrm{kHz}$. Considering that the $\mathrm{CF}$ is lower for humans than for cats [5], the results presented in this paper show a correlation between the highest DPOAE suppression, $Q_{10}$ reduction and the largest MOC innervation within the range of $2 \mathrm{kHz}$.

It is necessary to take into consideration the differences in the type of CS used. In this study the contralateral signal was a wideband noise, however Vinay and Moore [5] used a band of noise as the CS. Using broadband CS seems to be justified because with measurements of OAE this type of CS provides the greatest suppression effect $[14,25]$.

This research used the 'fast method' of measuring PTC because it yields comparable results to the classic method [39]. To test the effect of the method of measuring PTC has on the results, the author is going to carry out an investigation of wideband CS by using the classic method for determining PTC.

\section{Conclusions}

This study presents the results of research into DPOAEs and PTCs in the presence of wideband noise contralateral stimulation. The results allow the following conclusions to be drawn:

- wideband noise contralateral stimulation has a significant effect $(F(1,9)=19.36, p=0.002)$ on decreasing the value of $Q_{10 \text { Roex }}$ of PTCs,

- the largest decrease in the value of $Q_{10 \text { Roex }}$ occurs for test tone frequency of $2 \mathrm{kHz}$,

- the slope on the low-frequency sides of PTC $\left(\mathrm{p}_{\text {LRoex }}\right)$ significantly decreases $(F(1,9)=6.052$, $p=0.015)$ as the effect of CS, 
- frequency position of the minimum of the psychophysical tuning curve $\left(\operatorname{Min}_{\mathrm{PTC}}\right)$ is not significantly change under the influence of CS $(F(1,9)=$ $0.011, p=0.919)$,

- CS caused a decrease in the level of the DP component (suppression effect) in $88 \%$ of cases, in the whole $F_{2}$ frequency range (from $845 \mathrm{~Hz}$ to $6202 \mathrm{~Hz}$ ).

\section{Acknowledgments}

This work was supported by a grant from the National Science Center No. N N518 405438 Part of this study was also presented at the 164th Meeting of the Acoustical Society of America, Kansas City 2012.

\section{References}

[1] M.C. Liberman, Hear. Res. 34, 179 (1988).

[2] S. Chery Croze, E. Veuillet, L. Collet, A. Morgon, Rev. Laryngol. Otol. Rhinol. 114, 113 (1993).

[3] J.J. Guinan, Jr., M.L. Gifford, Hear. Res. 33, 97 (1988).

[4] J.J. Guinan, Jr., Ear Hear. 27, 589 (2006).

[5] Vinay, B.C. Moore, Hear. Res. 240, 93 (2008).

[6] G. Lisowska, G. Namyslowski, M. Misiolek, W. Scierski, B. Orecka, E. Czecior, A. Dziendziel, Otolaryngol. Pol. 62, 747 (2008).

[7] E. Ozimek, A. Wicher, in: XXVIII International Congress of Audiology, Insbruck (Austria), 2006, p. 43.

[8] S. Henin, S. Thompson, S. Abdelrazeq, G.R. Long, J. Acoust. Soc. Am. 129, 2068 (2011).

[9] A. Wicher, Acta Phys. Pol. A 121, 78 (2012).

[10] A. Wicher, in: 164th Meeting of the Acoustical Society of America, Kansas City (USA), 2012, p. 2049.

[11] L. Robles, M.A. Ruggero, Physiol. Rev 81, 1305 (2001).

[12] G. Lisowska, J. Smurzynski, K. Morawski, G. Namyslowski, R. Probst, Acta Otolaryngol. 122, 613 (2002).

[13] F. Zhang, F.A. Boettcher, X.M. Sun, Int. J. Audiol. 46, 187 (2007).

[14] S.R. Atcherson, M.J. Martin, R. Lintvedt, Neurosci. Lett 438, 107 (2008).

[15] N.A. Francis, J.J. Guinan, Jr., Hear. Res. 267, 36 (2010).

[16] U.A. Kumar, C.S. Vanaja, Ear. Hear. 25, 142 (2004).

[17] S. Kim, R.D. Frisina, D.R. Frisina, Speech Comm. 48, 855 (2006).

[18] W. Wagner, K. Frey, G. Heppelmann, S.K. Plontke, H.P. Zenner, Acta Otolaryngol. 128, 53 (2008).

[19] R. Probst, B.L. Lonsbury-Martin, G.K. Martin, J. Acoust. Soc. Am. 89, 2027 (1991).

[20] E. Ozimek, A. Wicher, W. Szyfter, E. Szymiec, J. Acoust. Soc. Am. 119, 527 (2006).

[21] M. Pankowska, D. Hojan-Jezierska, E. Skrodzka, E. Szymiec, L. Kubisz, T. Świdzinski, A. Wicher, Acta Phys. Pol. A 119, 1035 (2011).

[22] A.L. James, R.J. Mount, R.V. Harrison, Clin. Otolaryngol. 27, 106 (2002).
[23] D.W. Purcell, B.E. Butler, T.J. Saunders, P. Allen, J. Acoust. Soc. Am. 124, 2133 (2008).

[24] S. Chery-Croze, A. Moulin, L. Collet, Hear. Res. 68, 53 (1993).

[25] D.S. Velenovsky, T.J. Glattke, Hear. Res 164, 39 (2002).

[26] X.M. Sun, J. Acoust. Soc. Am 123, 4310 (2008).

[27] C. Abdala, S.K. Mishra, T.L. Williams, J. Acoust. Soc. Am 125, 1584 (2008).

[28] R. Deeter, R. Abel, L. Calandruccio, S. Dhar, J. Acoust. Soc. Am 126, 2413 (2009).

[29] S.G. Kujawa, T.J. Glattke, M. Fallon, R.P. Bobbin, Hear. Res. 68, 97 (1993).

[30] T. Kawase, M. Ogura, H. Hidaka, N. Sasaki, Y. Suzuki, T. Takasaka, Hear. Res. 142, 63 (2000).

[31] N. Quaranta, A. Scaringi, S. Nahum, A. Quaranta, Acta Otolaryngol. 125, 520 (2005).

[32] H. Fletcher, Rev. Mod. Phys. 12, 47 (1940).

[33] D.D. Greenwood, J. Acoust. Soc. Am 33, 1344 (1961).

[34] D.D. Greenwood, J. Acoust. Soc. Am. 33, 484 (1961).

[35] E. Skrodzka, D. Hojan-Jezierska, Acta Phys. Pol. A 118, 155 (2010).

[36] T. Kawase, H. Hidaka, T. Takasaka, Hear. Res. 108 , 37 (1997).

[37] C.T. Grimes, H.N. Wright, J. Speech and Hear. Res. 25, 394 (1982).

[38] K. Kluk, B.C. Moore, Hear. Res. 194, 118 (2004).

[39] A. Sęk, J. Alcantara, B.C. Moore, K. Kluk, A. Wicher, Int. J. Audiol. 44, 408 (2005).

[40] A. Sęk, A. Wicher, S. Drgas, Archiv. Acoust. 32, 707 (2007).

[41] A. Wicher, E. Ozimek, E. Szymiec, Acta Phys. Pol. A 118, 179 (2010).

[42] D. Hojan-Jezierska, A. Olejnik, Acta Phys. Pol. A 118, 71 (2010).

[43] A. Hoekstra, R.J. Ritsma, in: Psychophysics and Physiology of Hearing, Eds. E.F. Evans, J.P. Wilson, Academic, London 1977, p. 263.

[44] B.C. Moore, J.I. Alcantara, Ear. Hear. 22, 268 (2001).

[45] B.C. Moore, B.R. Glasberg, B. Roberts, J. Acoust. Soc. Am 76, 1057 (1984).

[46] A. Sęk, B.C. Moore, Int. J. Audiol. 50, 237 (2011).

[47] B.R. Glasberg, B.C. Moore, Hear. Res. 47, 103 (1990).

[48] J. Muller, T. Janssen, G. Heppelmann, W. Wagner, J. Acoust. Soc. Am 118, 3747 (2005).

[49] W. Wagner, G. Heppelmann, J. Muller, T. Janssen, H.P. Zenner, Hear. Res. 223, 83 (2007).

[50] W. Lilaonitkul, J.J. Guinan, Jr., J. Neurophysiol 101, 1394 (2009)

[51] B.C. Moore, J. Acoust. Soc. Am. 63, 524 (1978).

[52] B.C. Moore, B.R. Glasberg, Hear. Res. 28, 209 (1987).

[53] M.C. Liberman, J. Neurophysiol. 60, 1779 (1988). 